\title{
Velocity Profile Pictographs of Amoeboid Movement
}

\author{
Robert A. Rinaldi \\ Department of Zoology and Entomology, The University of \\ Tennessee, Knoxville, Tennessee, U.S.A.
}

Received July 2, 1963

\section{Introduction}

Velocity profiles of protoplasmic movement are a simple and useful method in assessing the dynamics of protoplasmic streaming and amoeboid motion. In order to obtain a velocity profile one measures the velocity of normal granular inclusions in adjacent layers of plasmasol (flowing protoplasm) through the relatively non-moving plasmagel tube. Kamiya and Kuroda (1958), by single frame analyses of a motion picture film of streaming in Physarum polycephalum, reported that the centrally moving plasmasol was moving faster than the more laterally moving plasmasol, and the shape of this velocity profile was a truncated parabola. The parabolic velocity profile of Physarum is similar to the motion of a structurally viscous fluid forced by pressure through a capillary tube. Likewise, Allen and Roslansky (1959) found a parabolic velocity profile exhibited by the plasmasol of the amoeba, Chaos chaos.

In contrast, Kamiya and Kuroda (1956) discovered a different velocity profile in Nitella. The speed of the plasmasol, in this case, is greatest near the non-moving plasmagel. Therefore, they concluded the velocity profile of Nitella supports the possibility of an active sliding of the plasmasol over plasmagel, in a manner suggestive of a current theory of muscle contraction (Huxley and Hanson 1954).

Motion in an amoeba can be graphed by another method, termed the pictograph technique (Rinaldi and Jahn 1962), which records granular motion over a period of time in a single photograph. The granules of the amoeba, under darkfield illumination, appear as white spots. Upon selecting a proper exposure time any motion of these granules will record as white streaks on the photographic film. Granules that are moving rapidly register as long streaks, those moving less rapidly as medium sized streaks, while those not moving appear as dots.

The present report depicts motion by the pictograph technique and attempts to assess this recorded data with some recent contributions to the study of protoplasmic movement. For reviews and discussions of the numerous theories recently advanced to explain protoplasmic motion the reader is referred to Kamiya (1959), Allen (1961), Abé (1961), Wolpert and O'Neill (1962), Kavanaugh (1962), and Rinaldi and Jahn (1963). 


\section{Materials and methods}

Amoeba proteus was cultured according to the methods of Prescott and James (1955). In order to obtain a complete graph of an amoeba in a single pictograph the organisms were bisected with microneedles. Before bisection the amoebae were transferred to a food deficient medium for 48 hours to reduce the number of food vacuoles. The bisected organisms were then mounted under a petrolatum supported coverslip, but an air bubble was included to prevent anoxia and reduce pressure effects. The nucleate halves moved normally and were selected for these experiments.

The experimental apparatus was simple: 1. a bright illuminating source (Bausch and Lomb Carbon Arc Lamp), 2. a microscope with a darkfield condenser (American Optical Company), and 3. camera (35 $\mathrm{mm}$ Leitz Wetzlar).

Some limitations of the pictograph method are:

1. The streaks of the granules can be recorded as more than their actual length. This occurs when one streak overlaps another within the selected time interval. But when these streaks overlap, there is an indication of it by a brighter region of the streaks, for two white streaks superimposed on each other tend to reinforce each other's image on the film.

2. Those granules which are moving perpendicular to the film would record as shorter streaks than actual. In flowing protoplasm this is rare, as plasmasol usually flows in a laminar manner, but it does occur. Examination of such streaks reveals that they curve, or that their recorded intensity along the length of the streak is not uniform, and this tends to identify them.

3. There is a problem associated with the determination of the direction of movement of the organism during the time of exposure to the photographic film. The latter limitation is almost completely resolved by:

a. immediate study of the amoeba before and after the exposure.

b. keeping the time exposure relatively short (one or two seconds).

The pictographs are made by the organism, and these graphs are complicated. The pictographs record motion, and the moving organism is a three dimensional entity. Thus, whithin any portion of the pictograph (except at the lateral edges) there will exist granules moving rapidly in the centrally moving plasmasol, while in the same region the motionless granules of the plasmagel tube will also record. The author will attempt to emphasize some of the more easily interpretable phenomena of amoeboid motion depicted in the graphs. But it is assumed that the reader, upon studying the pictographs, will discover additional facets of information concerning the motion exhibited by the amoeba.

\section{Results}

Velocity profiles of flowing plasmasol: Fig. 1 is an amoeba which has been photographed for one second at low power magnification. The labeled 
dash lines were added to this pictograph as a convenient reference for describing motion of the granules in the amoeba. The line graph to the right of the amoeba pictograph is also presented to aid the reader in observing some of the data.

Generally the centrally moving plasmasol stream is suggestive of a river as it winds from the tail to the tip of the amoeba. In A-A near the tip of the advancing amoeboid cell all the granules at the advancing tip region are
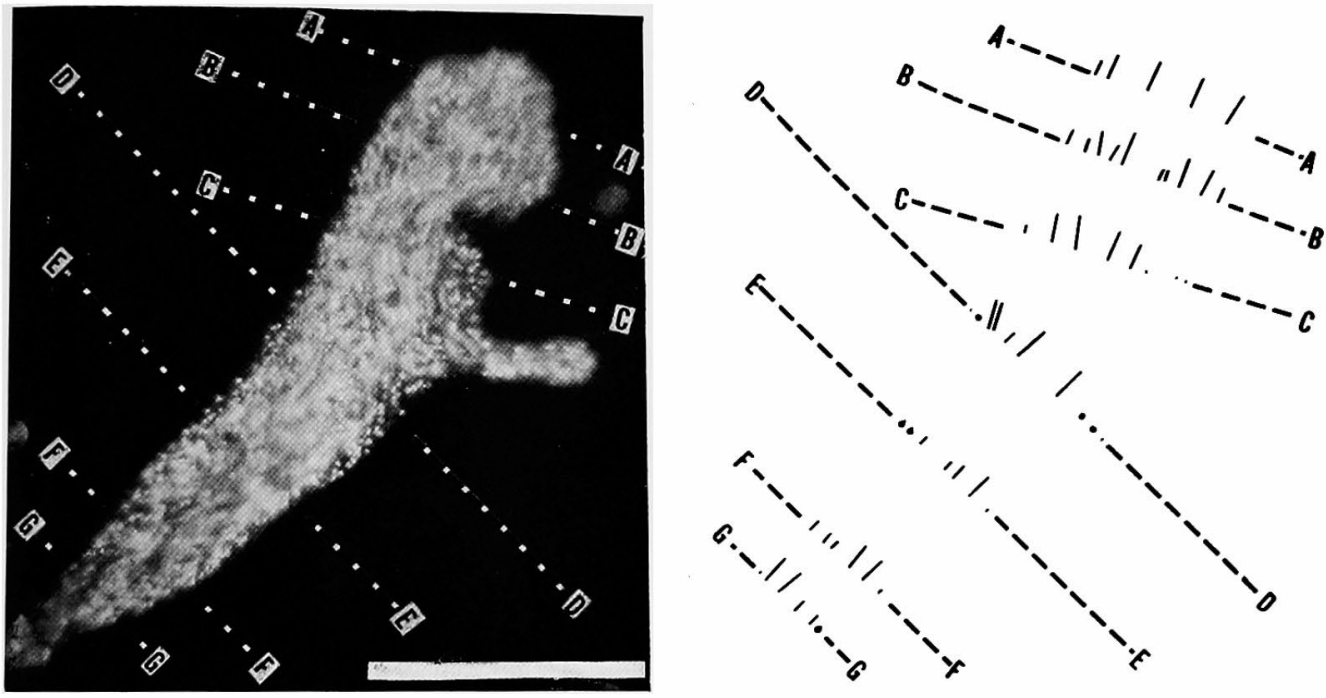

Fig. 1. Pictograph of an advancing amoeba, demonstrating the dynamics of flow in the plasmasol and the pseudopodial tip. The amoeba is advancing towards the upper right corner of the pictograph. The white bar is $125 \mu$ in length.

moving forward. Observe the parabolic velocity profile of the granules past A-A. In B-B there exists a region in the middle of the stream where the protoplasm is not moving so rapidly as the protoplasm near the edges as indicated by the small streaks in the center of the stream. In C-C, there is relatively uniform motion in the plasmasol and, for the first time, one observes distinct gelated regions along the sides which are indicated by the lack of streaking of the granules. From this point (C-C) to the pseudopodial tip, a distance of about 60 to $70 \mu$, all of the granules are moving, since there are no granules which record as dots. Further, there is a lack of backward deflection of granules recorded in this pictograph.

Observe, there is not an equal amount of gel on the right side of $\mathrm{D}$ as compared to the left side of $\mathrm{D}$. The values are approximately $15 \mu$ of plasmagel on the right to $5 \mu$ plasmagel on the left. An interference of the flowing granules in the central stream occurs at D near the side. This interference to flow graphs as "crescent" shaped streaks. Note in E-E and F-F that the central granules along the left side are flowing forward much more slowly than the granules on the right side; however, in G-G the opposite is true. 
The amoeba pictographs in Fig. 2 are one second exposures taken in sequence of a single moving amoeba with approximately 5-10 second intervals between each exposure. Again one observes a general flow of plasmasol through a gelated tube. The gelated tube is non-moving, and its granules record as dots or slight streaks; it occupies the region between the two arrows (placed in each pictograph). The $\mathrm{X}$ and $\mathrm{Y}$ axes are indicated by the dash line; they were drawn relative to "markers" which are usually present on the microscope slide.

The tail region: This extends from the lower left-hand side of the pictograph of each amoeba to the first arrow. The tail has moved forward approximately $35 \mu$ in pictograph 2 as compared to pictograph 1 . In pictograph 3 the tail region has advanced forward, relative to its environment, another $10 \mu$. But a large amount of streaking is evident in all graphs, and the length of the streaking in the pictographs indicates a movement of approximately $80 \mu$. The granules leave long streaks which are greater in their length than the tail's entire forward motion (pictograph 1 to pictograph 3 ). Streaking of the granules records only during the actual exposure of the film; however, there is a time between each exposure from 5 to 10 seconds during which the granules continue to move. But the net forward motion of the tail can be depicted accurately by merely comparing pictograph 1 to pictograph 3 ; therefore, there is an even greater motion of the granules from the tail region than indicated in the pictographs. This acceleration of the granules from the tail region probably represents a basic aspect of amoeboid motion and will be reviewed in the discussion section.

The plasmagel tube region: This occupies about $350 \mu$ and its position does not change substantially in the three pictopraphs even though the amoeba is advancing forward (to the right). Notice in distinct contrast to the plasmagel tube the rapidly flowing plasmasol. This flowing plasmasol is well indicated by the large vacuole lying directly below the dash line of the $\mathrm{X}$ axis in pictograph 1 ; in pictograph 2 the vacuole is directly under the dash line of the $\mathrm{X}$ axis, while in pictograph 3 the vacuole is located immediately above the $\mathrm{X}$ axis line. This vacuole has moved (pictograph 1 compared to pictograph 3) $140 \mu$. One may conclude from the data in this region that there is a nonmoving plasmagel tube through which the plasmasol rapidly flows.

The anterior region: The anterior region is defined as extending from the extreme tip of the advancing pseudopod (upper right-hand corner) to the arrow nearest the tip of the pseudopod. In pictograph 1 the anterior region occupies approximately $360 \mu$. In pictograph 2 the anterior tip has moved forward a distance of $90 \mu$, and the entire region is now $450 \mu$ in length. In pictograph 3 the tip of the anterior region has moved forward another $75 \mu$, and now the anterior region occupies $525 \mu$ of the pseudopod.

There is a "bending" motion of the anterior region towards the $\mathrm{X}$ axis. The white spot interrupting the dash line of the $\mathrm{Y}$ axis is used as a reference 

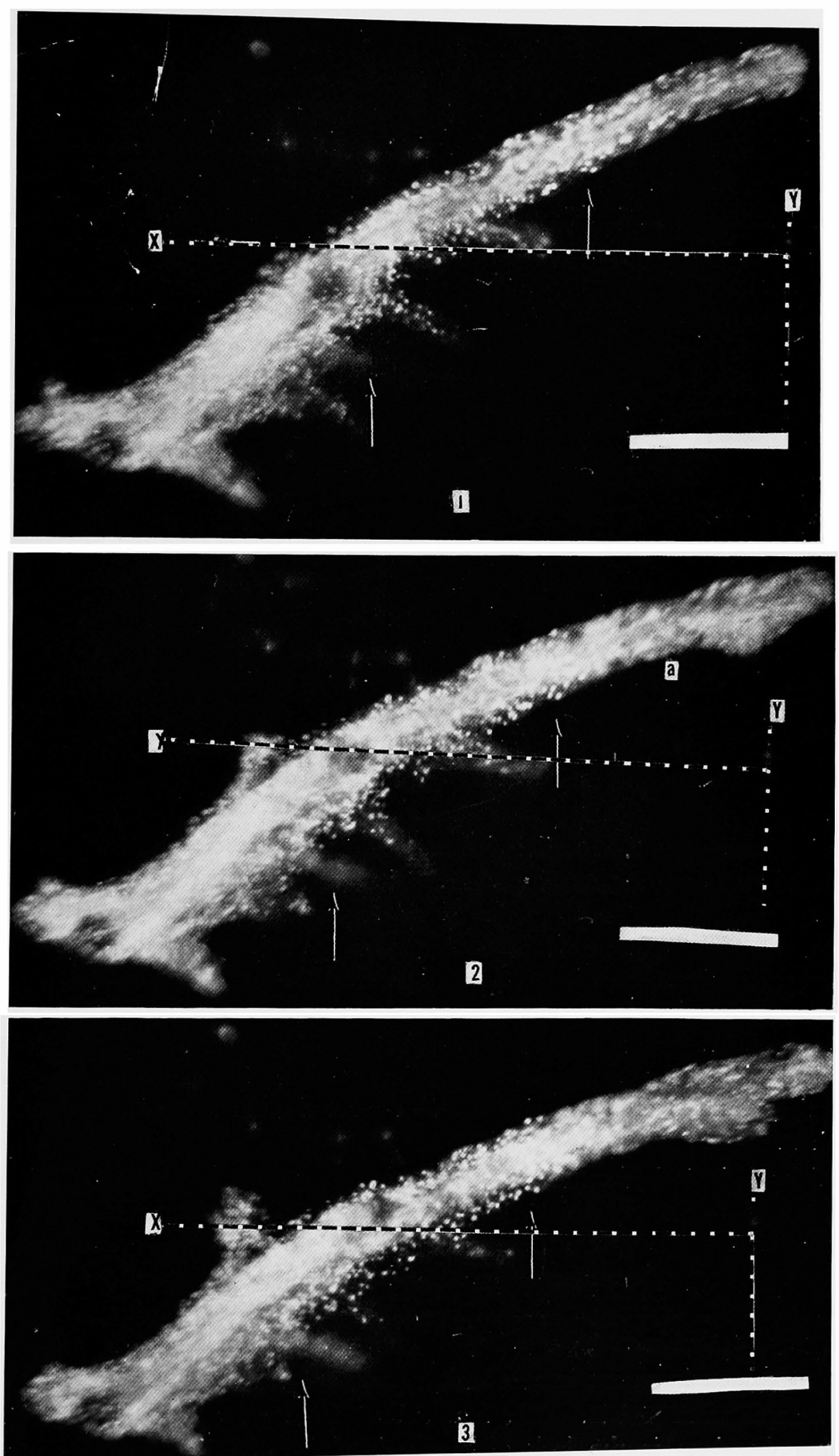

Fig. 2. Three pictographs taken in sequence of the same advancing amoeba. The pictographs are of one second duration, and the time lapse between each pictograph is approximately 5 to 10 seconds. Dash lines indicate the $\mathrm{X}$ and $\mathrm{Y}$ axes. The white bar in the lower right-hand corner is $250 \mu$ in length. 
point for this downward motion. The extreme top of the pseudopod is $325 \mu$ from this reference point, while in the second pictograph it is $285 \mu$ from this marker, and in pictograph 3 it is only $270 \mu$ from this reference point. Therefore, in this sequence the advancing pseudopodial tip has deflected $55 \mu$ towards the $\mathrm{X}$ axis.

The tail region, other than the advancing extreme posterior portion, has hardly moved at all towards the $\mathrm{X}$ axis. The point of reference to the $\mathrm{X}$ axis is the base of the small projecting pseudopod to the right near the tail region. Further, the angle at which the tail region projects toward the $\mathrm{X}$ axis has not changed substantially. This lack of change in the angle of the gel tube in the posterior region indicates that there is no bending or twisting of the plasmagel tube in this region.

Observe the striking torsion region exhibited by the "plasmagel" tube (directly above a) in the anterior advancing region. One cannot strictly apply the term plasmagel as used by Mast (1926), since there exist two phase of moving protoplasm here, so technically both phases should be plasmasol; therefore, to avoid additional terminology, the slower moving protoplasm in contrast to the more rapidly forward flowing protoplasm in the central region will be termed the "plasmagel" tube. Note the three granules (pictograph 1) at the tip of the arrow in this torsion region. In the second pictograph, their angle has changed relative to the axis of flow, about 40 degrees in comparison to the first pictograph; whereas in the third pictograph, the three granules have returned to a parallel position relative to the main channel of flow.

Fundamentally, the streaks of these granules in this torsion zone are the result of two components of motion. They are traveling back towards the tail region and down towards the $\mathrm{X}$ axis. In pictograph 2 the streaks in the "plasmagel" tube zone record as a vectorial sum of the above two components of motion. Relative to the environment the "plasmagel" tube moves backward toward the tail region approximately $30 \mu$ while, as indicated previously, the advancing tip region is bending down towards the $\mathrm{X}$ axis $(55 \mu)$. In pictograph 3 the backward motion has stopped, in comparison to figure 2 , as the granules of this region are in almost the same position as in pictograph 2.

Morphological phenomenon: In order to examine the "contractions" of the plasmagel tube, and to emphasize the movements, outline drawings (Fig. 3) were made of the three pictographs. The technique was to outline the amoeba with a wax pencil on cellophane, and then compare the shapes and movements of the amoeba as recorded over a period of time by the pictograph technique. Two points relative to the $\mathrm{X}$ and $\mathrm{Y}$ axis, which represent the environment in the background, are placed within the outline of the amoeba at $\mathrm{A}$ and $\mathrm{B}$. One fact gained from an examination of these outline drawings is the lack of change in shape of this amoeba during its 
locomotion. There is a slight decrease in girth near the posterior end, and an expansion of the plasmagel tube is evident near the anterior region. There is the extension of a new pseudopod (labeled 6) in the amoeba. But, other than these changes, there is a relatively constant morphology exhibited by this amoeba during its locomotion over a time interval for this advancing organism of 20 to 45 seconds.

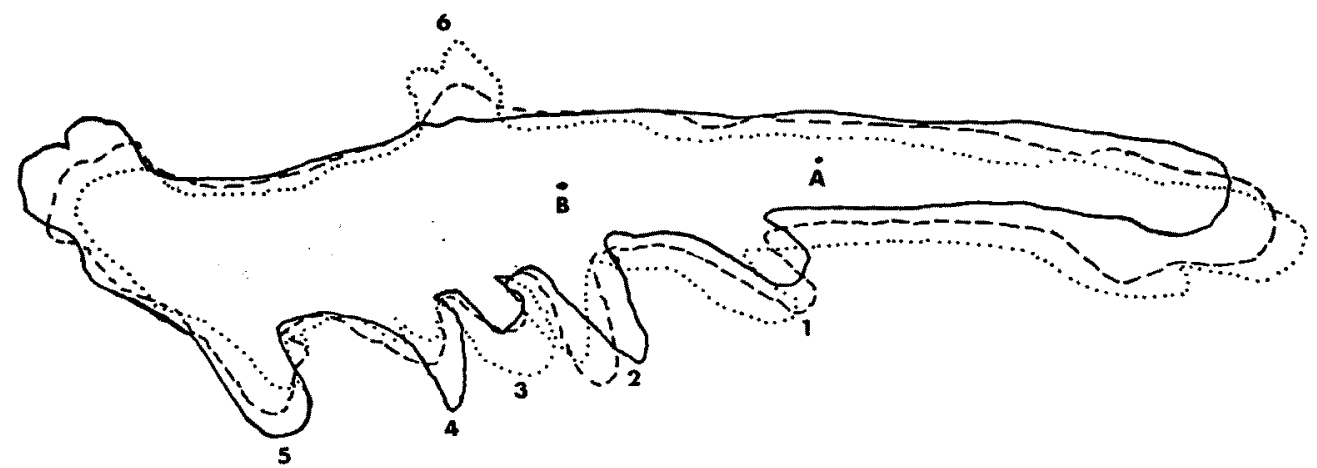

Fig. 3. Outline drawing of the amoeba in Fig. 2 emphasizing the changes in shape of the organism as well as its displacement during locomotion. The solid line is an outline of the first pictograph, the dash line is the second pictograph, and the dotted line is an outline drawing of the third pictograph.

An analysis of this outlines will re-emphasize some of the data noted previously. Observe the flow of plasmasol forward at the advancing tip as the amoeba moves forward. The deflection of the anterior region toward the $\mathrm{X}$ axis is quite noticeable. The first prominent pseudopod (labeled 1) has moved backwards during forward locomotion of the amoeba. However, the second pseudopod (labeled 2) starts to move backward when one compares the outline of pictograph 2 to pictograph 1 . But in pictograph 3 compared to pictograph 2 the position of this pseudopod is the same and obviously the backward motion has stopped. Pseudopods 3,4 and 5 and the forming pseudopod (labeled 6) have not changed or altered their positions relative to the amoeba's motion in the evironment (other than lengthening and/or shortening) during the recorded movement of this amoeba.

\section{Discussion}

The velocity profiles obtained in this study of plasmasol of Amoeba proteus are interpreted as being in complete agreement with the results reported by Kamiya and Kuroda (1958) for Physarum, and by Allen and Roslansky (1959) for Chaos chaos. There were different flow patterns on one side of the amoeba relative to the other side which are in agreement with the report by Allen and Roslansky (1959). There do exist some differences, but these are interpreted as due to the nature and states of the protoplasmic gelations and solations as well as the complexity associated with "pressure" being expressed on a non-Newtonian fluid such as protoplasm. 
In Fig. 2, three pictographs of the same amoeba were taken sequentially in order to graph movements of the organism, but it is emphasized that there exists one major limitation of these data, i.e. the time interval of five to ten seconds between each pictograph during which motion continues. In spite of this limitation, the data concerning the plasmagel tube are both impressive and fundamental. First, the plasmagel tube is capable of contraction. This is indicated by a portion of the "plasmagel" tube moving backwards in the anterior region, yet "holding" its position more posteriorly. This backward motion of the "plasmagel" tube (torsion zone) does not appear to be due to a pulling of the plasmagel tube from the rear end of the amoeba, which is indicated by the lack of streaks in the plasmagel tube more posteriorly. There is simultaneously a motion of the pseudopod downward. Evidently the component of motion towards the $\mathrm{X}$ axis is transporting the entire front end of the pseudopod downward.

This motion towards the $\mathrm{X}$ axis of the "plasmagel" tube could be explained by either a localized contraction of the "plasmagel" tube, or a secondary motion due to a force generated on the "plasmagel" tube by the rapidly flowing plasmasol. In this latter case, it is conceivable that there has become a restriction in free motion of the plasmasol forward, probably at a point of attachment of the "plasmagel" tube to the slide, resulting in an expansion immediately before this point of attachment. This bulging would tend to roll the "plasmagel" tube over because of pressure of the forward flowing plasmasol. But this latter possibility would not explain the backward motion of the "plasmagel" tube. From the evidence one can not state whether this torsion region is passive (results from plasmasol flow) or active (results from a contraction of the plasmagel). Contraction of the plasmagel tube is not surprising, and torsion either active or passive can be predicted from the classical theory of amoeboid locomotion advanced by Mast (1926).

It would seem proper to consider the changes in shape of an amoeba in order to test the consistent and repeated suggestion that changes in shape of Amoeba proteus are a foundamental prerequisite for amoeboid motion. The most surprising fact gained from a study of Fig. 3 is the lack of change in shape of the amoeba during its locomotion. There is a slight decrease in diameter of the plasmagel tube in the tail region, but this decrease can hardly be assessed in terms of possible protoplasmic progression, as one does not know the force which could be generated by a decrease in size of the elastic plasmagel tube. Further, even knowing the exact force produced by a decrease of the plasmagel tube, one would still be confronted by the problem of the effect of a known force on a complex fluid like plasmasol. Tentatively, one concludes that changes in shape may not be a main mechanism of amoeboid locomotion, and this conclusion supports the observations reported by Abé (1961), who noted that Amoeba striata during rapid locomotion exhibited no change in its shape. 
A fundamental part of this study of amoeboid motion is the impressive streaking in the tail region. This streaking is a record of an active acceleration of plasmasol from the advancing tail region of the amoeba. This acceleration, it is proposed, represents the motive force for amoeboid motion. But one must consider the question, what is meant by contraction of protoplasm? One established theory for muscle contraction, the sliding model theory of Huxley and Hanson (1954), requires an active sliding of a specific protein (actin) over another specific protein (myosin). This sliding model theory is generally accepted at the present time. Recently, actin and myosin have been found in Amoeba proteus; the distribution and arrangement of these proteins, however, are not established (Simard-Duquesne and Couillard 1962). It seems to be a safe assumption that the motive force for movement in a muscle cell and for movement of an amoeboid cell might have a similar basic mechanism.

Assuming that muscle cell contraction has the same physical and chemical parameters as the contraction of an amoeboid cell, then it could be proposed that the basic contractile event in an amoeba might be a dynamic active sliding of plasmasol on plasmagel. The greater streaking which occurs in the advancing tail region of the amoeboid cell reported in this paper supports this concept of an active movement of plasmasol from the interstices of the plasmagel tube. If the plasmasol is actively propelled from the plasmagel region, this could lead to a "collapsing" of the plasmagel tube, for one would assume that the plasmagel tube retains a degree of contractility (elasticity?). Removal of plasmasol by an active acceleration from the interstices of the gel region would decrease the support contributed by the plasmasol to the plasmagel tube and would tend to initiate the "collapse" of the plasmagel tube in specific regions. This "collapsing" of the plasmagel tube would contribute more force to the actively moving plasmasol and further aid in plasmasol motion. Moreover, it may well be that the "collapsing" of the plasmagel tube and the movement of plasmasol from the interstices of the plasmagel might lead to a localized "breakdown" of the plasmagel tube itself; this predicts the mechanics for conversion of plasmagel to plasmasol.

Plasmasol actively moving from the interstices of the plasmagel tube is a modification of Mast's theory of amoeboid motion, which was first suggested by Kamiya (1959) for Physarum. In addition, it is emphasized that this idea is not a simplified model, but it represents a dynamic interplay between plasmagel and plasmasol. This interplay reflects the morphology of the plasmasol and the plasmagel, the states of the plasmasol and the plasmagel, their dynamic interconversion, the kinetics of the plasmasol and the plasmagel, and finally their orientation to one another. This idea that an amoeboid organism may move by an active sliding of the plasmasol on the plasmagel remains speculative. Further evidence must be accumulated before one can more accurately 
determine those relationships which exist between the plasmagel and the plasmasol during amoeboid movement.

\section{Summary}

Graphs were obtained of granular motion in Amoeba proteus by the pictograph technique which records the motion exhibited by granules, illum. inated by darkfield microscopy, in a single time exposure photograph. The pictographs are made by the organism and are accurate but complex recordings. Information obtained from this method demonstrates that the plasmagel tube is capable of contraction. However, there is no significant change in morphology of this organism during its locomotion. Importance is placed on the active acceleration of plasmasol from the interstices of the tail's plasmagel. It is suggested that this might be the basic "contractile" event of amoeboid locomotion which supports the concept advanced by Kamiya for Physarum that an active sliding of plasmasol on plasmagel is a fundamental requirement of protoplasmic motion.

\section{Acknowledgments}

I wish to express appreciation to my colleagues in the Department of Zoology and Entomology at the University of Tennessee, Knoxville, for their kindness and aid during the course of this investigation. I wish to thank particularly Prof. Ronald C. Fraser and Prof. Arthur W. Jones for the loan of their personal equipment and for their criticisms of this work.

\section{Literature cited}

Abé, T. H. 1961. Morpho-physiological study of amoeboid movement. 1. Dynamic organization of striata amebae. Cytologia 26: 378-407.

Allen, R. D. and J. D. Roslansky 1959. The consistency of ameba cytoplasm and its bearing on the mechanism of ameboid movement. 1. An analysis of endoplasmic velocity profiles of Chaos chaos (L). J. Biophys. and Biochem. Cytol. 6: 437-446.

- 1961. Ameboid movement. In The Cell, vol. 2: ed. by Brachet, J. and A. Mirsky Academic Press, New York, 135-216.

Huxley, H. E. and J. Hanson. 1954. Changes in the cross-striations of muscle during contraction and stretch and their structural interpretation. Nature 173: 973-976.

Kamiya, N. and K. Kuroda. 1956. Velocity distribution of the protoplasmic streaming in Nitella cell. Bot. Mag. (Tokyo) 69: 544-554.

- and - 1958. Studies on the velocity distribution of the protoplasmic streaming in the myxomycete plasmodium. Protoplasma 49: 1-4.

- 1959. Protoplasmic streaming. Protoplasmatologia 8: 1-199.

Kavanaugh, J. L. 1962. Cytoplasmic streaming and non-equilibrium interfaces. Exptl. Cell Res. 27: 595-588.

Mast, S. O. 1926. Structure, movement, locomotion, and stimulation in Amoeba. J. Morphol. 41: $347-425$.

Prescott, D. M. and T. W. James. 1955. The culturing of Amoeba proteus on Tetrahymena. Exptl. Cell Res. 8: 256-258. 
Rinaldi, R. A. and T. L. Jahn. 1962. Tracks of granules during amoeboid movement. J. Protozool. 9: (Suppl.) 6.

- and - 1963. On the mechanism of amoeboid movement. Accepted for publication in J. Protozool., 1963.

Simard-Duquesne, N. and P. Couillard. 1962. Ameboid movement II. Research of contractile proteins in Amoeba proteus. Exptl. Cell Res. 28: 92-98.

Wolpert, L. and C. H. O'Neill. 1962. Dynamics of the membrane of Amoeba proteus studies with labelled specific antibody. Nature 196: 1261-1266. 